\title{
Real-Time Monitoring and Alarm System in Underground Coal Mines Using Smart Helmets (A Case Study: Tabas Coal Mine)
}

\section{Daniyal Ghadyani}

Amirkabir University of Technology

Amirhossein Badraddini

Amirkabir University of Technology

Mohammad Mirzehi Kalateh Kazemi

Tarbiat Modares University

Vahab Sarfarazi ( $\square$ vahab.sarfarazi@gmail.com )

Hamedan University of Technology

Sohrab Naser Mostofi

Tarbiat Modares University

Vahid Khodabandeloo

Tehran University: University of Tehran

\section{Research}

Keywords: Underground mines, smart safety helmet, smart monitoring, wireless data transmission

Posted Date: October 12th, 2021

DOI: https://doi.org/10.21203/rs.3.rs-951389/v1

License: (1) This work is licensed under a Creative Commons Attribution 4.0 International License. Read Full License 


\section{Abstract}

Regarding the hazard-prone working conditions in underground mines, synchronous monitoring and alarm system is vital to increase the safety. By analyzing the accidents in underground mines in Iran, it can be deduced that most fatalities are related to harmful gas leakage, objects drop off on the head, and not using helmets by the staff. Therefore, a smart helmet with the capability of measuring harmful gasses (regarding the type of the mine), detection of the existence of the helmet on the head, temperature and humidity measurement, and detection of blow on the head is designed and fabricated to eliminate the present dangers and problems. This system displays the evaluated data on a developed software through wireless data transmission hardware. The data transmission hardware is the primary link between the intelligent safety helmet and the software. To follow the idea, practical experiments have been performed in Parvadeh four and East Parvadeh of Tabas coal mine to confirm the validity of data transmission that culminated in successful results. The results were altered by the complexity of the design of the underground spaces so that in a straight direction, data transmission was held until 430 meters. However, further progress was not possible due to tunnel limitations. Data transmission was reduced to 190 meters in access horizons with curvatures or tilts. According to present standards, some thresholds are defined for each of the mentioned cases such that alarm protocol is activated by exceeding these thresholds in critical circumstances. Then the helmet user and the software's operator will be informed of the occurred danger and will settle the problem.

\section{Introduction}

Considering the number of people engaged in mining directly, the high investment and operational costs of mining activities, the uncertainty of various mining units, and the dangerous nature of mining operations, increasing the safety of these people while working is an unavoidable issue. On the other hand, occupational hazards and the healthcare issues related to the activities of the mine workers are fundamental concerns of this industry. It is evident that the safety in open pit mines is more than in underground mines due to adequate light and fresh air access (Paul,2009). Regarding the complexity of geology, the structure of present non-continuities, and their direct influence on the type of stress distribution, the underground mines are more accident-prone. Unexpected accidents and existing dangers in underground mines could provide a stressful and dangerous workplace for workers and operational facilities that impose considerable life and financial threats. Hazards of working in underground mines require particular attention to all influential factors of safety due to the circumstances emerging from area constraints, environmental pollutants, geology complexities, inadequate light, etc.

The surveillance of the mining environment and the workers' health are considered essential parameters in mining. On the other hand, the workers tend to avoid bringing some safety equipment due to its weight, heat, and bothersome ergonomy. The current safety helmets are utilized to protect the workers' heads against the impacts caused by objects drop off or colliding with protuberances inside the tunnel. 
In mining complexes, monitoring and appropriate communications are fundamental requirements for correctly managing human and machinery resources. HSE administrators should be aware of situations with hazard potentials. Therefore, it is crucial to have a monitoring and surveillance system to acquire the evaluated data, transmit it to the control center, and make the most appropriate decision at the earliest possible moment. There are two types of communication networks for underground places: wired and wireless networks. The wired networks have the following drawbacks:

- High installation and commissioning costs

- Being damaged during accidents

- High maintenance costs

- Time-consuming installation and repairing

- Difficult and time-consuming diagnoses

Regarding the mentioned disadvantages, implementing a wired network doesn't have technical and economic justifications. On the other hand, wireless networks do not have the weaknesses of the wired networks. Still, they are also technically and economically defensible, acquiring features such as high speed, high safety, self-diagnosis, remarkable reliability, etc.

This project is a continuous, real-time monitoring and surveillance system based on wireless communication to increase safety by monitoring various parameters such as temperature, humidity, dangerous gasses, impact to the head, helmet wearing, and navigation. Moreover, in addition to mentioned parameters, this system can also detect helmet wearing and measure impact to the head. Alongside alarming the control room operator, the worker will also be informed of the sort of danger considering the predicted protocols. As a result, the proposed system will increase the workers' safety and efficiency of the mining operation.

\section{Research Background}

The workers' safety is generally threatened due to poisonous gasses that usually exist in underground mines. These gasses are not detectable without facilities. Therefore, Srivastava (2015) studied toxic gasses in critical areas and their effects on miners. He implemented a real-time monitoring system using a network of wireless sensors. This system controls environmental parameters like temperature, humidity, and several poisonous gasses. Furthermore, it possesses an alarm section in the helmet that informs the workers and the people around of the dangerous circumstance according to the type of the threatening danger and can confront the issue at the earliest possible moment. The system uses ZigBee technology to generate a wireless sensor network. This network conforms to the IEEE 802.15.4 standard that is suitable to work under rough working conditions. Kesavan, Balaji, Sivashankaran and Yogeshwaran (2013) designed a smart safety helmet using ZigBee technology to monitor hazardous gasses, helmet removal from the head, and alert protocol. The analyzed gasses are Carbon monoxide, Sulfur dioxide, and 
Nitrogen dioxide. Kumar and Reddy (2018) designed a smart safety helmet to monitor dangerous gasses based on ZigBee. In addition, a stick is implemented on the safety helmets to identify the workers using Infra-Red (IR) sensors and to ensure the helmet wearing. Then they evaluated the blow to the head using micro-electromechanical switch (MEMS) sensors. Behr, Kumar and Hancke (2016) proposed a smart helmet to reduce mine dangers by analyzing three parameters of air quality, blow to head, and helmet wearing detection. The air quality factor includes the evaluation of the density of dangerous gasses. Borkar and Baru (2018) suggested generating a safe network using smart helmets to analyze the environmental conditions around the workers and real-time data transmission. Chi et al. (2014) described the relation for reconfigurable smart sensors for WSN industries on the Internet of Things (IOT) platform to gather all sensor data thoroughly. This system is designed based on the IEEE 1451 protocol. This system is totally convenient and fast to acquire real-time data quickly for the Internet of Things environment. Maity, Das and Mukherjee (2012) exhibited mineral parameters like unusual gasses, temperature, and humidity on a system to prevent the presence of dangerous gasses and high environmental temperature to workers. Hermanus (2007) investigated the performance of Occupational Health and Safety (OHS) against variations in the compositions of different sections in the mineral division of South Africa. They resulted that mining in South African suffers from a shortage of mutual interactions between workers and engineers, risk management education, and other things like these. Misra, Kanhere, Ostry and Jha (2010) described the common characteristics in a wide range of stressful environments, explained the factors that could affect communications while settling in these areas, and indicated the most challenging parameters in underground environments. Yarkan, Guzelgoz, Arslan and Murphy (2009) studied underground communications, general issues that underground communication systems must consider, and various types of communications, methods, and their significance. Chehri, Fortier and Tardif (2009) investigated and evaluated the UWB-based WSN navigation system with high accuracy in underground mines. The objective of these systems is to detect equipment and miners under regular operation or emergency conditions in the galleries of the underground mines. Hong Jiang and Shuangyou (2008) designed a real-time monitoring and alarm system based on CAN-BUS technology for underground areas and acquired data. S3C2410 microprocessor and ZigBee are implemented in this system as the central core of the system and wireless sensor network to transmit data, respectively. AbuMahfouz and Hancke (2013) introduced a navigation algorithm based on intelligent selection. This method only selects references that cause the navigation accuracy to be increased and minimizes the number of required iterations to correct the validity of the estimated location. Previous researches were reported the same results ((Lee 2013; Ruan 2015; Zhou 2018; Zhang 2021; Park 2021; Eick 2021; Ruccolo 2021; Borlenghi 2021).

The represented system in this research guarantees the performance of the system, relying on its alarm package. The novel insight is the detailed analysis of blow to head factor and converting it to the other factors by studying the contents related to the topic and bounding this factor instead of a number to judge. Also, the most transparent part of this design is increasing the managerial approach that added attendance evaluation platform and performance reporting to the system. 


\section{System Architecture}

This project is a persistent monitoring system with the capability of monitoring parameters like:

- the presence of toxic gasses (methane, liquid gas, and Carbon monoxide)

- temperature and humidity

- helmet wearing detection

- has the damage to the head scale.

The assessed values are transferred into the processor, and the processing operation is performed on the data. The processor compares the input data with the threshold limits (according to the present standards) and, by occurring unexpected unnatural conditions, activates the alarm protocol, including informing the control room operator and activating the alarm section installed on the helmet. This system includes safety helmets, data transmission, and monitoring sections. In the present set, the helmet plays the role of evaluating and transferring data to the data transmission section. The data transmission section provides the communication link between the safety helmet and the control room. In what follows, each section is discussed in more detail.

\subsection{Safety Helmet Section}

The structure of the safety helmet is demonstrated in figure 1 . This section is generally responsible for increasing safety (that is obvious), evaluation, processing, and transferring of data. The smart safety helmet has three subsections of evaluations, data transmission, and alarm. The responsibility and performance of these subsections are discussed in the following.

\subsubsection{Evaluation Subsection}

This subsection has the responsibility of monitoring and includes DHT11, CNY70, MQ9, LM35, and ADXL345 sensors that are temperature and humidity, infra-red, gas detection, temperature, and accelerometer sensors, respectively. The type and method of operation by these sensors are described in what follows:

\section{A) Gas measuring:}

In underground mines, air pollution is caused by methane gas, sulfur dioxide, nitrogen oxides, carbon monoxide, and also hovering particles. When a person is exposed to toxic gases for a long time, it could cause physical damages. MQ9 sensors are utilized to measure liquid gases in this project. MQ9 gas sensor has a high sensitivity to carbon monoxide, methane, and LPG. This sensor is fabricated from a ceramic AL2O3 micro-pipe and a sensitive layer of tin dioxide ( $\mathrm{SnO2}$ ) using a plastic grid and anticorrosion steel (Kumar and Hancke, 2014). The detection performance of this sensor is through temperature and humidity variations, and the rising density of the gas increases the conductivity of the 
sensor. These sensors detect carbon monoxide gas while the temperature is falling. As the temperature is rising, this sensor detects gasses of methane, propane, and other flammable gasses (Kumar and Hancke, 2014). As mentioned before, the sensor above has different responses to the density of gasses in various temperatures and humidities. Therefore, some temperature and humidity sensors must be applied at the proximity of these sensors.

Figure 2 represents the index of sensitivity of the MQ9 sensor in 20 degrees Celsius, $65 \%$ humidity, and oxygen density of $21 \%$. As it is evident from the figure, the sensor's resistance has different responses for three kinds of gasses, including carbon monoxide, methane, and liquid gas. It is worth mentioning that .these details are programmed by coding in the processor of the helmet and the developed software

B) Helmet wearing detection

IR sensors are utilized to recognize whether a worker is wearing a helmet or not. The mentioned sensor is composed of a transmitter and a receiver. The IR transmitter is a light-emitting diode radiating IR rays. Although IR lamps look like a regular Light Emitting Diode (LED), the radiated wave is invisible to human eyes. IR receivers are renowned as IR sensors as well because they detect radiation from an IR transmitter such that an IR wave is illuminated on the head while wearing a helmet, and the receiver of this ray does not acquire any data. Otherwise, it is realized that the helmet is not worn after propagation of the wave and receiver's acquisition (Hermanus, 2007).In addition, to increase the assurance of helmet wearing, IR sensors and accelerometers are implemented simultaneously. It could be deduced that the human head has a particular acceleration range by doing numerous experiments in various modes such as stationary, moving, etc. As a result, in case of not wearing the helmet, if the measured acceleration by accelerometer remains unchanged, wearing or not wearing the helmet would be detected. One of the other anticipated applications of these sensors is reporting the personnel's working hours and attendance performance.

C) Blow to head detection

According to the Federal Motor Vehicle Safety Standard 208 (FMVSS 208), a scale is defined with the symbol of HIC to detect the blow to the head, which should have values not exceeding 1000 . This value is calculated using equation (1) (Chou, Song and Lim, 1997), (forooshani, Bashir, Michelso and Noghanian, 2013), (Laksari et al, 2020), (Davis et al, 2000), (Saboori, Mansoor-Baghaei and Sadegh, 2013), (Thombare, 2019) and (Hutchinson, Kaiser and Lankarani, 1998).

$H I C\left(\Delta t_{\max }\right)=\max \left[\left(\frac{1}{t_{2}-t_{1}} \int_{t_{1}}^{t_{2}} \hat{a} d t\right)^{2.5}\left(t_{2}-t_{1}\right)\right]$

The damage scale is known as a measuring scale. The severity of the damage could be evaluated using the Abbreviated Injury Scale (AIS) that is varied from AIS(0), meaning no damage up to AIS(6), meaning unavoidable damage (forooshani, Bashir, Michelso and Noghanian (2013). In order to validate and calibrate the relation between the injury scale and the damage to head scale, some experiments should be performed under certain circumstances. HIC is a damage scale related to a skull fracture (AIS $\geq 2$ ) and 
brain injury (AIS $\geq 4$ ). The conversion from HIC into damage scale could be achieved by referring to the data plotted on figures 3 and 4 that are represented according to the experiments by Mertz, Prasad and Irwin (1997) to detect the presence or absence of skull fracture and intracranial hemorrhage. Furthermore, it is noted that the accelerometer produces wrong measurements. To overcome this design issue and compensate for it, the accelerometer is placed in the helmet rather than its plastic tape, which holds the head (Newman, 1975).

D) Temperature and humidity detection

The lack of appropriate ventilation in underground mines leads to temperature and humidity rise in these spaces that could cause reduced efficiency of the production process. Therefore, real-time and continuous monitoring of the temperature and humidity of the workplace is an unavoidable issue. On the other hand, to measure the density of the gasses precisely, the need for temperature and humidity sensors is obvious.

The implemented temperature sensor in this project is $\mathrm{DHT} 11$, one of the accurate temperature and humidity sensors with an analog output with a linear dependence on the environment's temperature in degrees Celsius. One of the advantages of this sensor is measuring the temperature on a Celsius scale, while most sensors work on the Kelvin scale. In addition, the humidity measured and represented by this sensor is in the form of relative humidity. This sensor does not need further calibrations (Ahalya, Babu and Rao, 2013).

\subsubsection{Data Transmission Subsection}

After processing data acquired from the evaluation subsection, the processor must send these data to the control room for monitoring and decision making. So, in order to transmit mentioned data, we decided to use a radio module. The processor is the core of the project because it processes the evaluated data from sensors and communicates with the data transmission subsection according to its predefined responsibilities (Fisher, Ledwaba, Hancke and Kruger, 2015). The ZigBee module is a wireless chip signals of which are capable of penetrating the walls and conforming to underground mine circumstances. The standard ZigBee module has a digital interface that allows every processor or microprocessor to rapidly use this protocol's services. ZigBee's performance is conformed to the IEEE 802.15.4 standard (Hongjiang and Shuangyou, 2008). ZigBee systems could be programmed to work in various network topologies like the star, light mesh, mesh, and clustered tree, four of the most applicable structures. Temporal networking structures are used where a node should send the information to all other nodes.

\subsubsection{Alarm subsection}

Alerting the mineworkers is a tricky process due to working conditions. There is not enough light in the space of underground mines, so the workers use safety helmets with replaceable mineral lamps. The utilized equipment generates too much noise and vibrations due to space limitations. The alarm 
subsection is composed of vibration, alarm buzzer, and LED units to overcome the mentioned problems. The considered protocol for alarm subsection is capable of responding in various conditions.

\subsection{Monitoring Subsection}

After acquiring the processed data, all of this information will be transferred into a software through a wireless network platform. The provided software has the duties of on-time detection of the occurred dangers and activation of the relief and rescue protocols. Figure 5 shows the developed software.

\section{Performance Style}

At first, an identification number is assigned to the helmet of every person that facilitates attendance evaluation, identification of working people in workstations, and conforming to personal hygiene. It is worth mentioning that to do data transmission experiments in mines, the Tabas coal mines are selected, and initial data transmission experiments have been performed there successfully.

\subsection{Introduction to Tabas coal mines}

The Parvadeh region is one of the coal regions of Tabas, with an area of 1200 square kilometers and coal layers extending over 40 kilometers. This extension, along with the continuity of coal layers in the whole region, is unique and enables the design of numerous mines in their proximities. Figure 6 demonstrates the geographical location of the Parvadeh region related to Tehran (the capital of Iran) and Birjand (the center of the province South Khorasan) (Ahangaran et al, 2011).

The Parvadeh region includes Parvadeh No. 1, Parvadeh No. 2, Parvadeh No. 3, Parvadeh No. 4, Parvadeh No. 5, and East Parvadeh. The coal regions are demonstrated in figure 7. In this article, the data transmission experiment is implemented in two areas of Parvadeh 4 and East Parvadehh.

Parvadeh No. 4 deposit is located 85 kilometers south of Tabas city. The coal in Kansar Parvadeh No. 4 is generated in a large synclinal along with shale rocks, sandstones, siltstones, and upper Triassic Basin carbonate stones. Regarding geological and sedimentology observations, it seems these stone units have sedimented in a free marine environment. The coals from Parvadeh No.4 deposit are bituminous coking coals with high sulfur ratios (Yazdi and Shirvavani, 2004).

East Parvadeh No.4 deposit is located 120 kilometers southeast of Tabas city. East Parvadeh is in the eastern part of the coal region of Parvadeh after Parvadeh No. 4 region. The area has a smooth topography, and high mountains do not exist (Ahangaran et al, 2011). The tunnels openings are through two central ramp accesses. Both tunnels start with a 12-degree slope inside the layer and continue with a slope of 16 degrees. One of the tunnels is equipped with a conveyor for hauling purposes, and the other is designed for various services of the mine using a winch (IRITEC, 1992).

\subsection{Designed Radio Module}


A wireless communication system simply consists of a transmitter and a receiver. The responsibility of the transmitter is to convert the considered message to an electric signal and then convert the electric signal to radio waves. At the destination, the receiver is responsible for acquiring waves and converting them into electric signals, amplifying them, and finally extracting the considered data from them. Radio communication systems work in different frequencies that are categorized as represented in Table 1 (Bandyopadhyay, Chaulya and Mishra, 2010).

Table 1

Different frequencies of radio communications (Bandyopadhyay, Chaulya and Mishra, 2010).

\begin{tabular}{|lll|}
\hline $3 \mathrm{KHz}-30 \mathrm{KHz}$ & VLF & Very Low Frequency \\
\hline $30 \mathrm{KHz}-300 \mathrm{KHz}$ & LF & Low Frequency \\
$300 \mathrm{KHz}-3 \mathrm{MHz}$ & MF & Medium Frequency \\
$3 \mathrm{MHz}-30 \mathrm{MHz}$ & HF & High Frequency \\
\hline $30 \mathrm{MHz}-300 \mathrm{MHz}$ & VHF & Very High Frequency \\
\hline $300 \mathrm{MHz}-3 \mathrm{GHz}$ & UHF & Ultra High Frequency \\
\hline $3 \mathrm{GHz}-30 \mathrm{GHz}$ & SHF & Super High Frequency \\
\hline$>30 \mathrm{GHz}$ & EHF & Extreme High Frequency \\
\hline
\end{tabular}

Each of the frequency bands is utilized for particular applications based on its feature. For instance, marine communication is generally performed in lower frequencies such as VLF. At the same time, terrestrial communications are in higher frequencies like FM radio in VHF (88 108MHz), television signals $(600 \sim 700 \mathrm{MHz})$, Mobile $(900 \mathrm{MHz}$ and $1800 \mathrm{MHz})$, and Bluetooth-based and WiFi-based systems $(2.4 \mathrm{GHz})$. In general, the waves with frequencies equal to or higher than $1 \mathrm{GHz}$ are called Microwaves, and the waves with frequencies equal to or higher than $30 \mathrm{GHz}$ are called Millimeter waves (Bandyopadhyay, Chaulya and Mishra, 2010). Therefore, to resolve one of the primary requirements of this project in underground mines that is data transmission, an infrastructure is provided in this project using a communication platform in the UHF band. Figure 8 represents the radio module. Using this module in Tabas coal mines, a data transmission test has been designed and performed. Regarding the tilts in the main tunnels and access horizons, data transmission is successfully accomplished by a 190-meter transmission in one horizon with four inclinations and no direct vision and a 430-meter transmission in the main slope, including three ramps of $12 \%, 14 \%$, and $16 \%$.

The software has a page called "report" responsible for continuous data acquisition and uploading the data in a cloud storage space. Figures 9 and 10 exhibit the sent reports using a smart safety helmet and a plot of mine. According to the standards mentioned earlier, the safety zones for each factor are measured. In case of exceeding the predefined threshold by any factor, the factor is indicated in red in the software. 
Finally, the most important managerial section is performance reporting. Figures 9 and 10 provide a unified representation of the performance report that indicates the working time in green and the stopping time (for any reason) in red.

\section{Conclusion}

A real-time monitoring and alarm system is essential for detecting dangerous accidents such as the presence of harmful gasses, helmet removal by workers, object drop off on the head of a worker, and transmitting data to the relevant administrators. Therefore, the smart safety helmet is successfully designed and tested for the mining industry to detect and register dangerous events. Appropriate hardware is implemented precisely for each of the considered performances of the safety helmet based on the current standards and by relying on the performed researches. The recent endeavors help to improve the performance of the system. The installation of this system is quick and simple and has high reliability. The result of all these actions is graphically implemented and displayed in the user interface. The user can make the required decisions using the developed software in different conditions. One of the other outputs of the system is the conversion of the blow to the head into the damage scale, which could facilitate and speed up the rescue effort to the damaged person in critical circumstances.

\section{Declarations}

\section{Ethical statements}

- The authors have avoided for tests on the animals.

- The manuscript only has been submitted to this journal.

- The submitted work is original and have not been published elsewhere in any form or language

- This study has not been split up into several parts.

- Concurrent or secondary publication is sometimes justifiable, provided certain conditions are met. Examples include: translations or a manuscript that is intended for a different group of readers.

- Results was presented clearly, honestly, and without fabrication, falsification or inappropriate data

- No data, text, or theories by others have not been presented as if they were the author's own.

\section{References}

1. "hwsensor," HANWEI ELETRONICS CO., LTD, 2014. [Online]. Available: http://www.hwsensor.com. 2. Abu-Mahfouz, A. M., \& Hancke, G. P. (2013). An efficient distributed localisation algorithm for wireless sensor networks: based on smart reference-selection method. International Journal of Sensor Networks, 13(2), 94-111.

3. Ahalya, G., Babu, P. S., \& Rao, P. P. (2013). Development of coal mine safety system using wireless sensor networks. Intern. J. of Engineering Science \& Advanced Technology, 3(3), 74-78. 
4. Ahangaran, D. K., Afzal, P., Yasrebi, A. B., Wetherelt, A., Foster, P. J., \& Darestani, R. A. (2011). An evaluation of the quality of metallurgical coking coal seams within the north block of Eastern Parvadeh coal deposit, Tabas, Central Iran. Journal of Mining and Metallurgy A: Mining, 47(1), 9-24.

5. Borlenghi P., (2021) Detecting and localizing anomalies on masonry towers from low-cost vibration Monitoring. Smart Structures and Systems, An Int'I Journal, 27(2):88-99.

6. Bandyopadhyay LK, Chaulya SK, Mishra PK (2010). Wireless communication in underground mines. RFID-Based Sens. Netw, 22

7. Behr, C. J., Kumar, A., \& Hancke, G. P. (2016, March). A smart helmet for air quality and hazardous event detection for the mining industry. In 2016 IEEE International Conference on Industrial Technology (ICIT) (pp. 2026-2031). IEEE.

8. Borkar, S. P., \& Baru, V. B. (2018). loT based smart helmet for underground mines. International Journal of Research in Engineering, Science and Management (IJESM), 1(9).

9. Chehri, A., Fortier, P., \& Tardif, P. M. (2009). UWB-based sensor networks for localization in mining environments. Ad Hoc Networks, 7(5), 987-1000.

10. Chi, Q., Yan, H., Zhang, C., Pang, Z., \& Da Xu, L. (2014). A reconfigurable smart sensor interface for industrial WSN in IoT environment. IEEE transactions on industrial informatics, 10(2), 1417-1425.

11. Chou CC, Song GS, Lim GG (1997). Head Injury Criterion (HIC) Calculation Using an Optimization Approach (No. 971046). SAE Technical Paper

12. Davies JM, Wallace WA, Colton CL, Tomlin O, Payne AR (2000). The Head Injury Criteria and Future Accident Investigations

13. Eick B., (2021) Non-contact Monitoring of the tension in partially submerged, miter-gate diagonals Smart Structures and Systems, An Int'I Journal, 27(2):33-45.

14. Fisher, R., Ledwaba, L., Hancke, G., \& Kruger, C. (2015). Open hardware: A role to play in wireless sensor networks Sensors, 15(3), 6818-6844.

15. Forooshani, A. E., Bashir, S., Michelson, D. G., \& Noghanian, S. (2013). A survey of wireless communications and propagation modeling in underground mines. IEEE Communications surveys \& tutorials, 15(4), 1524-1545.

16. Hermanus, M. A. (2007). Occupational health and safety in mining-status, new developments, and concerns. Journal of the Southern African Institute of Mining and Metallurgy, 107(8), 531-538.

17. Hongjiang, H., \& Shuangyou, W. (2008, August). The application of ARM and ZigBee technology wireless networks in monitoring mine safety system. In 2008 ISECS International Colloquium on Computing, Communication, Control, and Management (Vol. 2, pp. 430-433). IEEE.

18. Hutchinson, J., Kaiser, M. J., \& Lankarani, H. M. (1998). The head injury criterion (HIC) functional. Applied mathematics and computation, 96(1), 1-16.

19. IRITEC, (1992). "Internal Reports of Tabas Coal Mine," pp 110-135, Tabas.

20. Kesavan, S., Balaji, E., Sivashankaran, K., \& Yogeshwaran, R. (2013). Smart Safety Helmet for Miners. South Asian Journal of Engineering and Technology, 3(4), 118-123. 
21. Kumar, A., \& Hancke, G. P. (2014). Energy efficient environment monitoring system based on the IEEE 802.15. 4 Standard for low cost requirements. IEEE Sensors Journal, 14(8), 2557-2566.

22. Lee S., Yun G., (2013) A statistical reference-free damage identification for Real-Time Monitoring of truss bridges using wavelet-based log likelihood ratios. Smart Structures and Systems, An Int'I Journal 12(2) 66-78.

23. Laksari, K., Fanton, M., Wu, L. C., Nguyen, T. H., Kurt, M., Giordano, C., ... Camarillo, D. B. (2020). Multidirectional dynamic model for traumatic brain injury detection. Journal of neurotrauma, 37(7), 982993.

24. Maity, T., Das, P. S., \& Mukherjee, M. (2012, March). A wireless surveillance and safety system for mine workers based on Zigbee. In 2012 1st International Conference on Recent Advances in Information Technology (RAIT) (pp. 148-151). IEEE.

25. Mertz, H. J., Prasad, P., \& Irwin, A. L. (1997). Injury risk curves for children and adults in frontal and rear collisions. SAE transactions, 3563-3580.

26. Misra, P., Kanhere, S., Ostry, D., \& Jha, S. (2010). Safety assurance and rescue communication systems in high-stress environments: A mining case study. IEEE Communications Magazine, 48(4), 66-73.

27. Newman JA (1975). On the use of the head injury criterion (HIC) in protective headgear evaluation (No. 751162). SAE Technical Paper

28. Paul, P. S. (2009). Predictors of work injury in underground mines-an application of a logistic regression model. Mining Science and Technology, 19(3), 282-289.

29. Ruan J., Zhang Z., (2015) An anti-noise Real-Time cross-correlation method for bolted joint Monitoring using piezoceramic transducers. Smart Structures and Systems, An Int'I Journal, 16(2):99-111.

30. Ruccolo A., (2021) Monitoring an iconic heritage structure with OMA: the Main Spire of the Milan Cathedral. Smart Structures and Systems, An Int'I Journal, 27(2), 66-77.

31. Park J., (2021) Development of wireless SHM sensor node for in-flight Real-Time Monitoring using embedded CNT fiber sensors. Smart Structures and Systems, An Int'I Journal, 28(3):44-57.

32. Ravi Kumar, G., \& Keerthi Reddy, B. (2018). Internet of Things Based an Intelligent Helmet for Wireless Sensor Network. International Journal of Engineering Sciences \& Research Technology (IJESRT).

33. Saboori P, Mansoor-Baghaei S, Sadegh AM (2013, November). Evaluation of head injury criteria under different impact loading. In ASME International Mechanical Engineering Congress and Exposition (Vol. 56215, p. V03AT03A076). American Society of Mechanical Engineers.

34. Srivastava, S. K. (2015). Real time monitoring system for mine safety using wireless sensor network (multi-gas detector) (Doctoral dissertation).

35. Thombare DG (2019). Computational and Experimental Analysis of Head Injury Criteria (HIC) in Frontal Collision of Car with Pedestrian (No. 2019-26-0016). SAE Technical Paper 
36. Yarkan, S., Guzelgoz, S., Arslan, H., \& Murphy, R. R. (2009). Underground mine communications: A survey. IEEE Communications Surveys \& Tutorials, 11(3), 125-142.

37. Yazdi, M., \& Shiravani, A. E. (2004). Geochemical properties of coals in the Lushan coalfield of Iran. International Journal of Coal Geology, 60(1), 73-79.

38. Zhou L., (2018) Real-Time condition assessment of railway tunnel deformation using an FBG-based Monitoring system. Smart Structures and Systems, An Int'l Journal, 21(5):111-123.

39. Zhang Q., (2021) Real-Time structural health Monitoring system based on streaming data. Qilin, Siyuan Sun, Bin Smart Structures and Systems, An Int'I Journal 28(2):77-89.

\section{Figures}

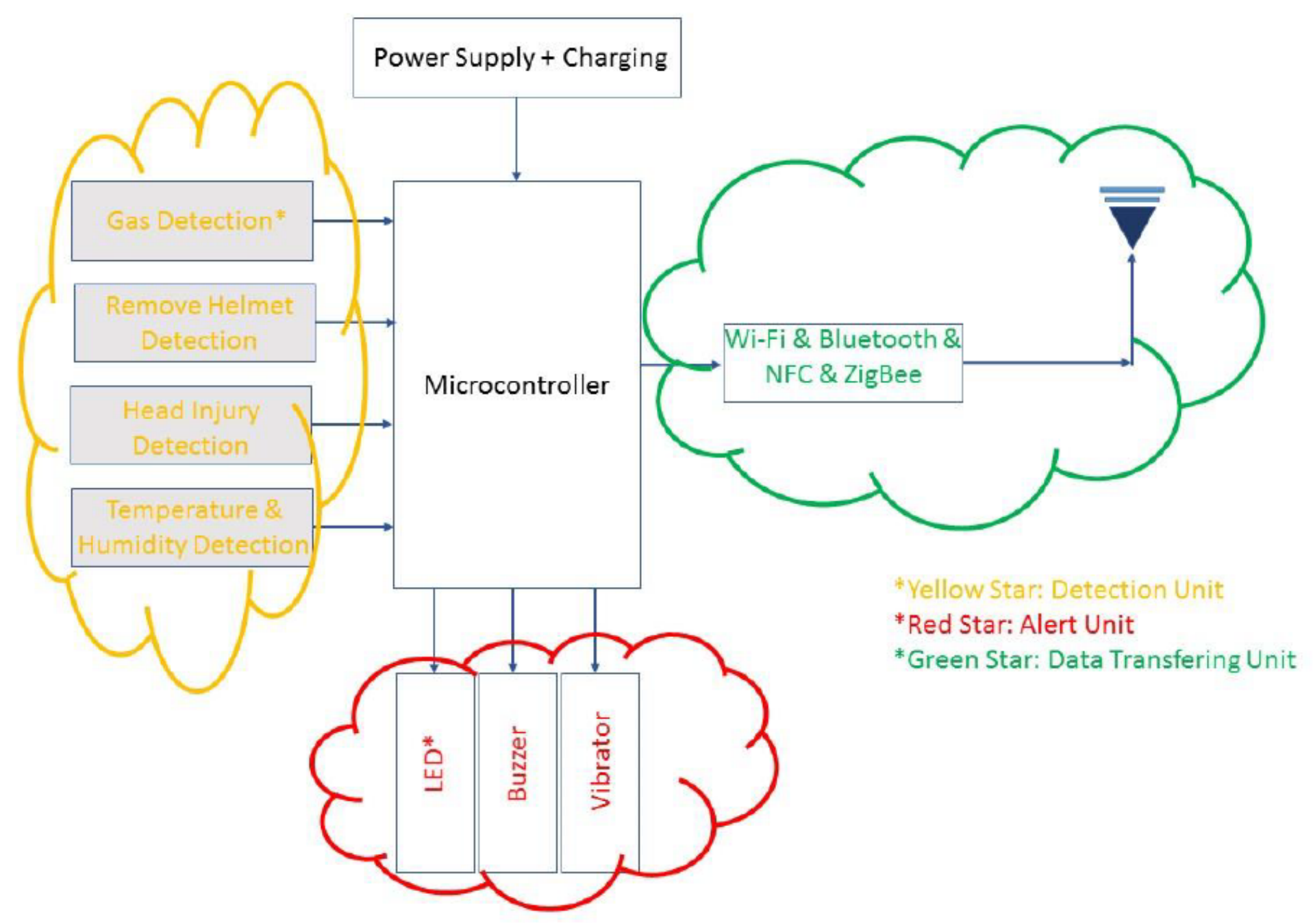

\section{Figure 1}

The architecture of the safety helmet. 


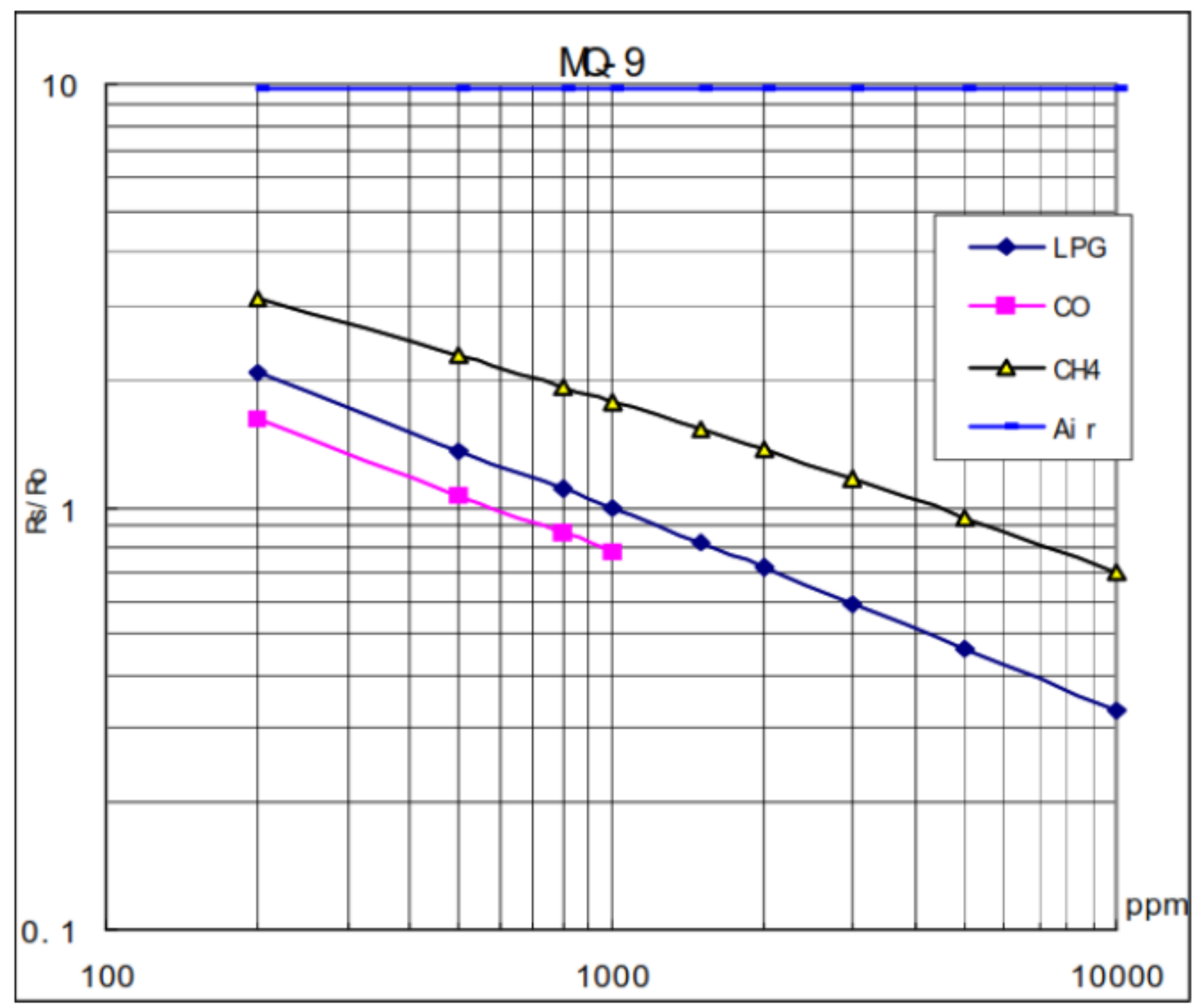

Figure 2

Sensitivity index of MQ9 sensor (hwsensor, 2014). 


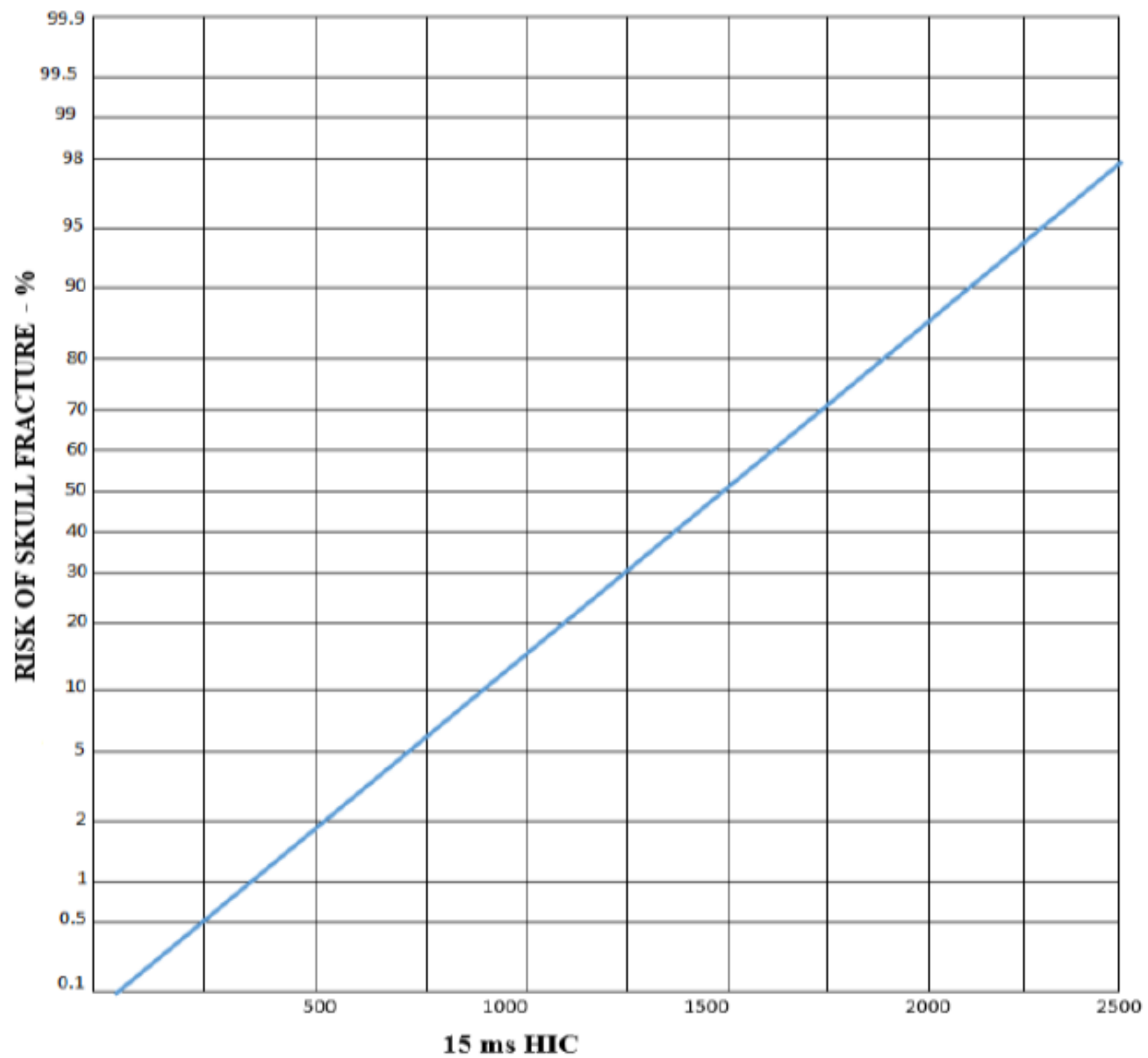

Figure 3

Skull damage scale as a function of HIC15 function (Mertz, Prasad and Irwin ,1997). 


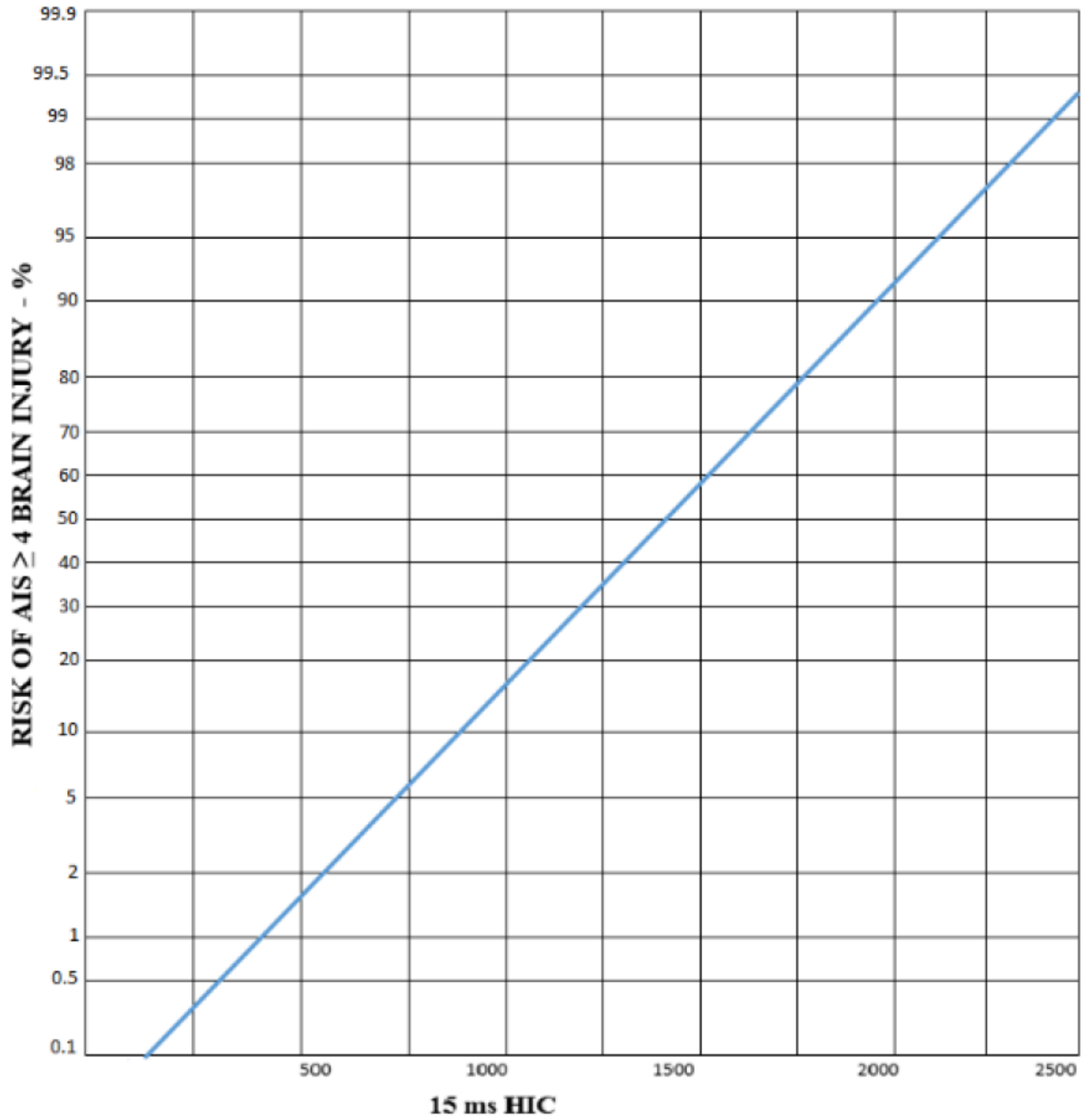

Figure 4

Brain injury scale as a function of HIC15 function (Mertz, Prasad and Irwin ,1997). 


\begin{tabular}{|c|c|c|c|c|c|c|c|c|c|c|}
\hline$\hat{\imath}$ & Battery Status (\%) & Humidity (\%) & Temp $\left({ }^{\circ} \mathrm{C}\right)$ & Density (ppm) & Gas type & HIC & Status & Floor & Name & No. \\
\hline & 100 & 21 & 25 & 658 & LPG & 335 & Resting & $1^{*}$ & Janiyal Ghadyani & 1 \\
\hline & 100 & 20 & 27 & 951 & LPG & 232 & Resting & $2^{\text {st }}$ & Vahid Ghasemi & 2 \\
\hline & 100 & $\cdots$ & 26 & 850 & LPG & 380 & Resting & $2^{a t}$ & Farshid Nazemi & 3 \\
\hline & 100 & 18 & 28 & 819 & LPG & 362 & Resting & $2^{a x}$ & Amir Abbaslou & 4 \\
\hline & 100 & 21 & 28 & 657 & LPG & 258 & Resting & $2^{a t}$ & Karim Eslami & 5 \\
\hline & 100 & 22 & 28 & 988 & LPG & 413 & Working & $2^{\text {st }}$ & Akbar Sadeghi & 6 \\
\hline & 100 & 17 & 28 & 799 & LPG & 304 & Working & $2^{\text {th }}$ & Ali Rajabi & 7 \\
\hline & 100 & 17 & 26 & 624 & LPG & 356 & Working & $2^{a t}$ & Sajad Najmi & 8 \\
\hline & 100 & 23 & 24 & 899 & LPG & 341 & Working & $2^{a t}$ & Naser Kazemi & 9 \\
\hline & 100 & $\cdots$ & $\cdots$ & $\cdots$ & $\cdots$ & $\cdots$ & Absent & $2^{t t}$ & Arash Momeni & 10 \\
\hline & 100 & 16 & 26 & 822 & LPG & 316 & Resting & $1^{\mathrm{s}}$ & Hossein Rezaei & 11 \\
\hline & 100 & 23 & 21 & 924 & LPG & 379 & Working & $1^{s t}$ & Hamid Hosseini & 12 \\
\hline & 100 & 21 & 28 & 965 & LPG & 218 & Working & $1^{\text {t }}$ & Saeid Najafi & 13 \\
\hline & 100 & 23 & 28 & 879 & LPG & 359 & Resting & $1^{\text {tt }}$ & Ali Rezvani & 14 \\
\hline & 100 & 23 & 25 & 851 & LPG & 482 & Kestıng & $1^{\text {t }}$ & Ali Mousavi & 15 \\
\hline & 100 & 18 & 26 & 717 & LPG & 332 & Resting & $1^{\text {th }}$ & Kazem Ahmadi & 16 \\
\hline & 100 & 16 & 29 & $\cdots$ & $\cdots$ & 299 & Working & $1^{\text {it }}$ & Majid Ahmad & 17 \\
\hline & 100 & $\cdots$ & $\cdots$ & $\cdots$ & $\ldots$ & $\cdots$ & Working & $3^{\text {tt }}$ & Soheil Ghasemi & 18 \\
\hline & 100 & 24 & 24 & 828 & LPG & 224 & Working & $2^{\text {at }}$ & Reza Hojjati & 19 \\
\hline
\end{tabular}

\section{Figure 5}

Status tab in the developed version of the software 


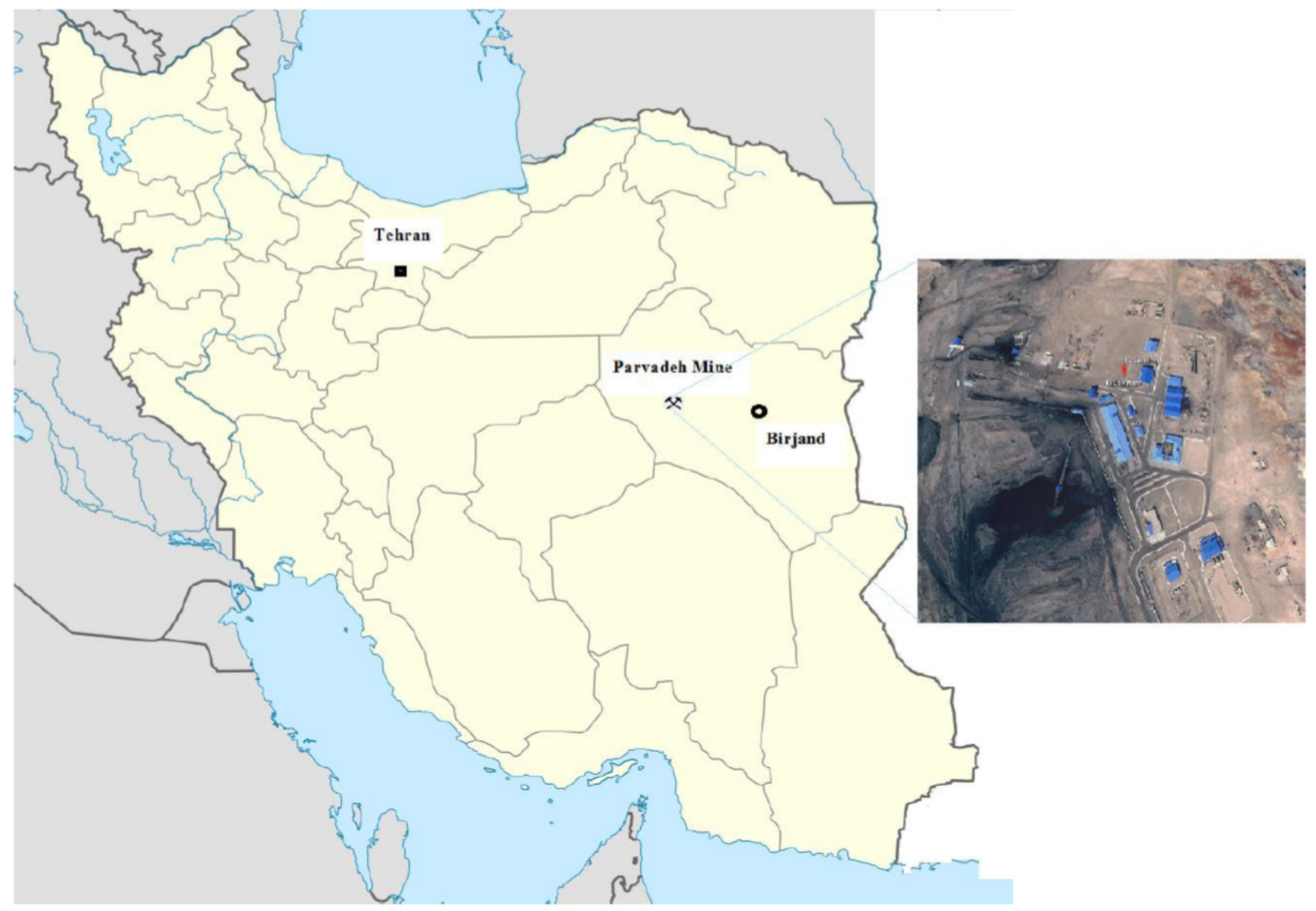

Figure 6

The geographical location of Parvadeh region relative to Tehran (the capital of Iran) and Birjand (the center of the province South Khorasan) ) (Ahangaran et al, 2011). 


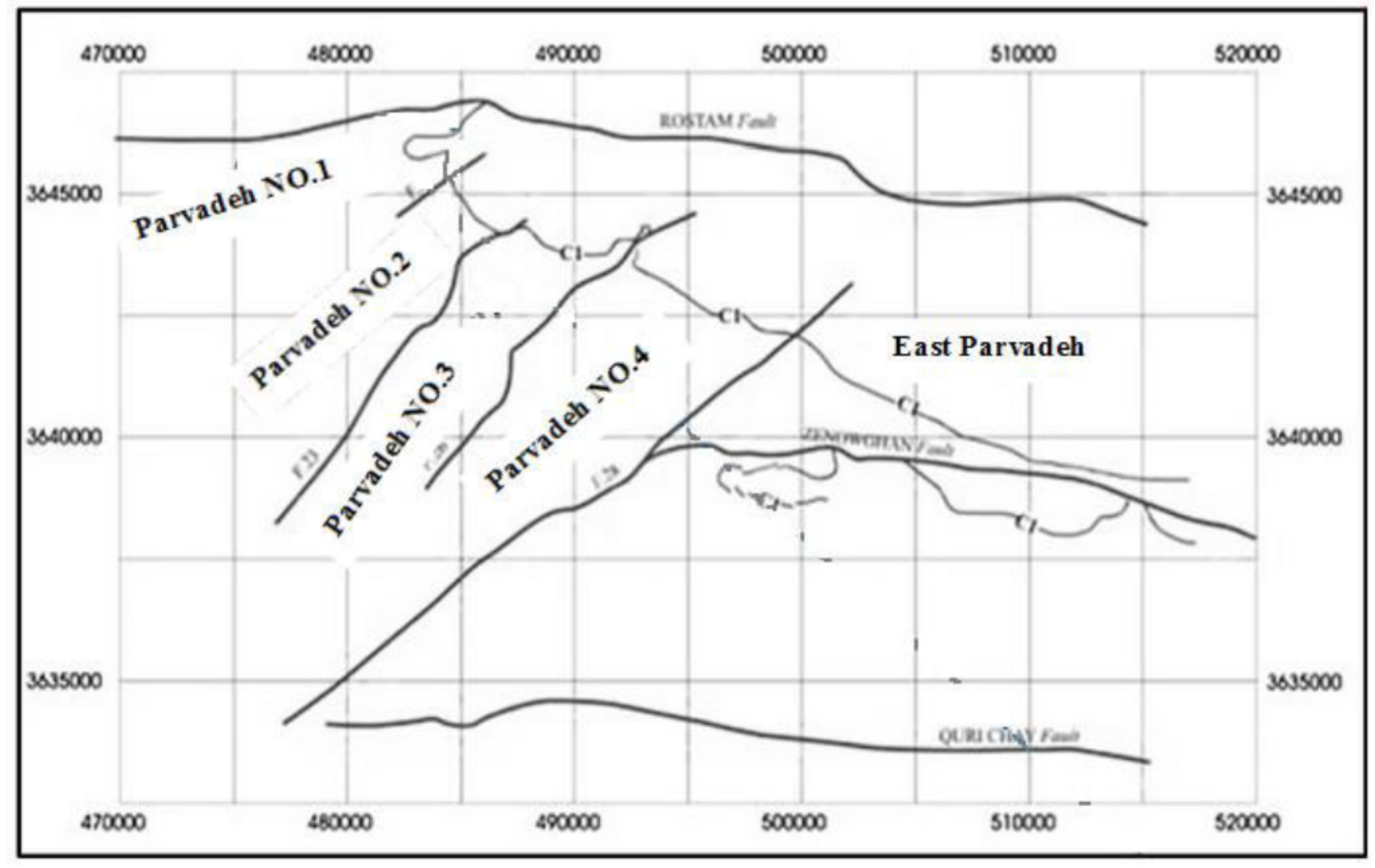

Figure 7

The plan of coal regions of Parvadeh area (with no scales) (Ahangaran et al, 2011). 


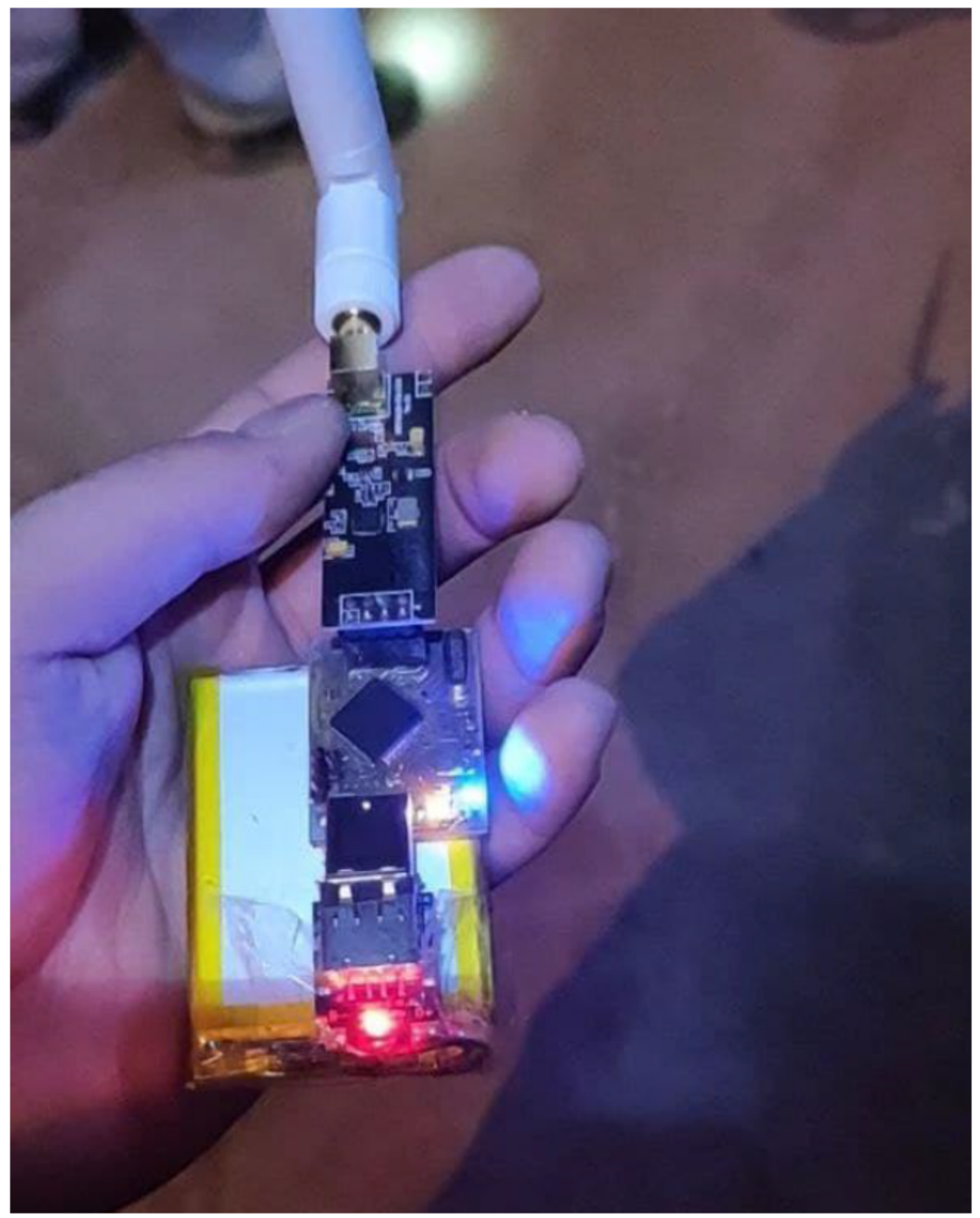

Figure 8

Designed radio module for testing data transmission in the tunnels of underground mines. 


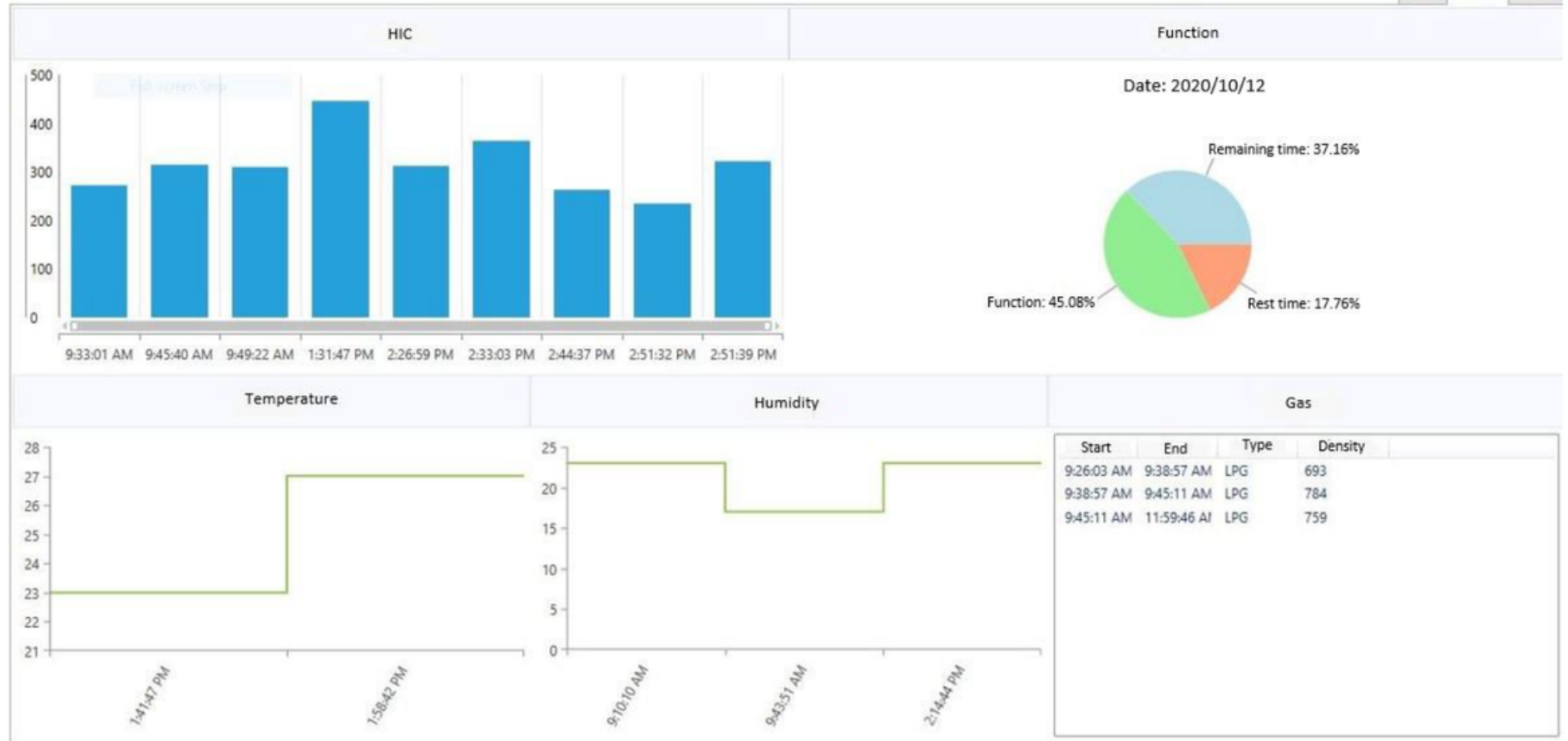

Figure 9

Graphical representation of typical data in the "Details" tab. 


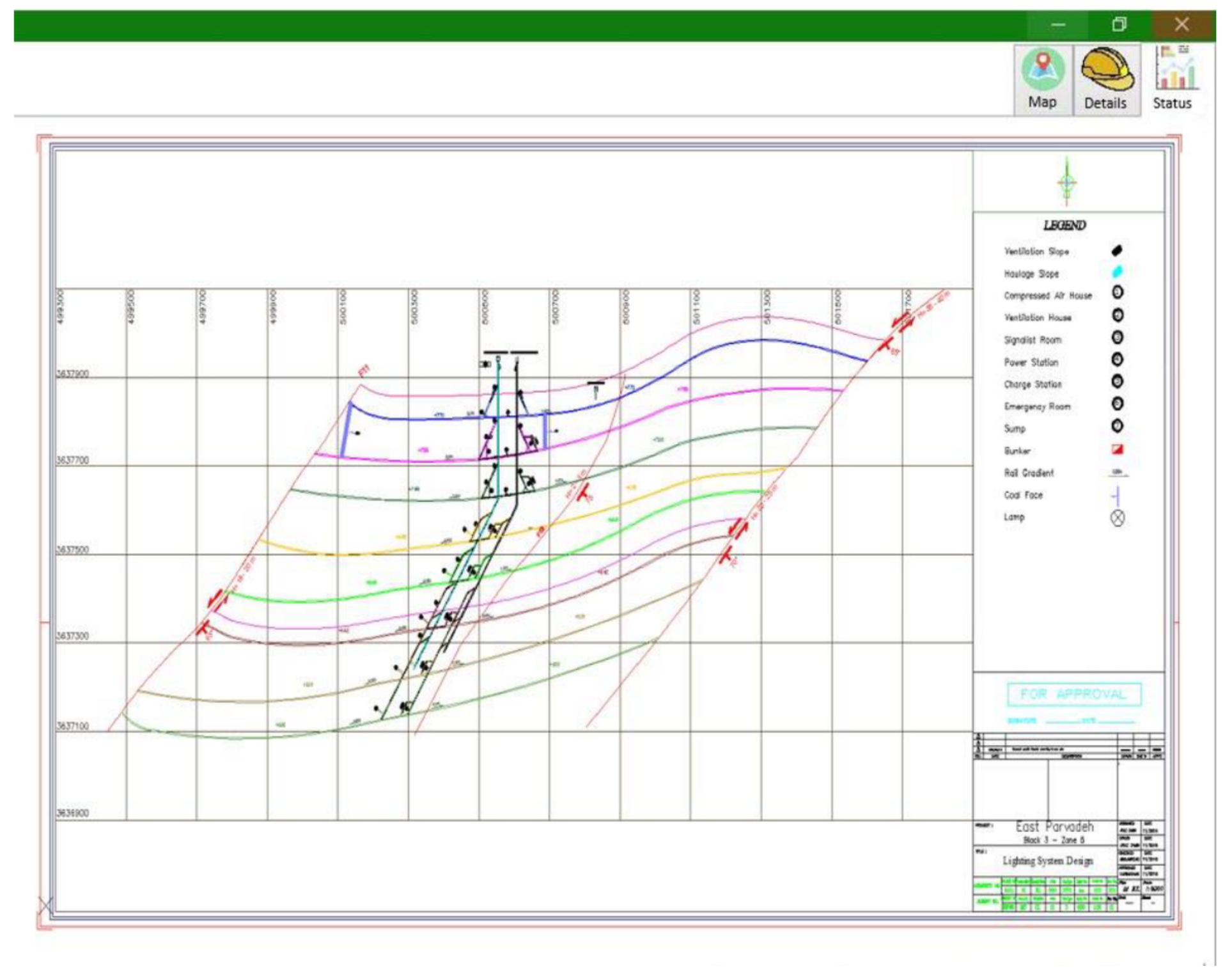

Figure 10

Graphical representation of a mine in the developed software. 